\title{
Tribune du président de la SSDV Forum des Präsidenten der SGDV
}

\section{Mesdames, Messieurs, Chers Collègues}

Le 7 février 2002 à Berne s'est déroulée une assemblée générale extraordinaire de la SSDV. Vous en trouverez ci-joint le procès-verbal. Les décisions suivantes ont été prises et les conséquences financières qui en résultent ont été acceptées:

- La SSDV se dotera dès sa prochaine assemblée générale en novembre 2002 d'un secrétaire général. Cette professionnalisation du fonctionnement de la société est acceptée pour une période de 3 ans et sera ensuite réévaluée.

- L'activité des membres de la société travaillant pour la SSDV est rétribuée selon un règlement d'indemnisation (proposition de modification des statuts ci-après).

- La SSDV reste membre de la FMS, pour une année, puis réévaluation.

Ces trois décisions ont comme conséquence que la cotisation 2002-2003 de la SSDV va augmenter de manière importante, soit passer à un montant d'environ CHF 1000,- plus les CHF 250,- de cotisation FMS si notre affiliation est maintenue pour 2003.

Vous trouverez ci-joint un document concernant le cahier des charges du secrétaire général de la SSDV. Cette activité est estimée à un poste de travail à $40 \%$. Une recherche active d'un candidat est faite dès maintenant et jusqu'à fin septembre 2002. Tout membre de la société intéressé à une telle fonction est prié d'ores et déjà de faire acte de candidature auprès du Président de la SSDV.

Tout membre de la société ayant des propositions de candidats qui rempliraient ce cahier des charges est prié d'en informer le Président.

En vous remerciant de votre précieuse collaboration, veuillez croire, Mesdames, Messieurs, Chers Collègues, à nos sentiments les meilleurs.

Prof. R.G. Panizzon

Président

Dr F. Gueissaz

Membre du Comité

\section{Liebe Kolleginnen und Kollegen}

Am 7. Februar 2002 hielten wir in Bern die ausserordentliche Generalversammlung der SGDV ab. Sie finden in der Beilage das Protokoll. Folgende Entscheide wurden vorgenommen und die daraus resultierenden finanziellen Konsequenzen angenommen:

- Die SGDV wird ab nächster Generalversammlung im November 2002 über einen Generalsekretär verfügen. Diese Professionalisierung der Geschäfte unserer Gesellschaft ist für eine Dauer von 3 Jahren angenommen und wird dann reevaluiert werden.

- Die Tätigkeit der Mitglieder, die für die SGDV arbeiten, wird gemäss einem Vergütungsreglement entschädigt werden (Vorschlag der Statutenänderungen nachfolgend).

- Die SGDV bleibt Mitglied der FMS, für die Dauer eines Jahres, dann erfolgt eine Reevaluation.

Diese drei Entscheide haben zur Folge, dass der Beitrag 2002-2003 der SGDV markant zunehmen wird, das heisst er wird ungefähr CHF 1000.- plus CHF 250.- als Beitrag für die FMS, falls wir Mitglied bis 2003 bleiben, ausmachen.

Sie finden in der Beilage ein Dokument betreffend das Pflichtenheft des Generalsekretärs der SGDV.

Der Arbeitsaufwand für diesen Posten beträgt etwa 40\%. Ab heute bis Ende September 2002 beginnt aktiv die Suche nach einem geeigneten Kandidaten. Mitglieder unserer Gesellschaft, die sich für einen solchen Posten interessieren, sind gebeten, sich als Kandidaten beim Präsidenten der SGDV zu melden.

Diejenigen Mitglieder unserer Gesellschaft, welche einen geeigneten Kandidaten vorschlagen wollen, sind gebeten, dies dem Präsidenten mitzuteilen.

Mit bestem Dank für Ihre Mitarbeit und mit freundlichen Grüssen.

Prof. R.G. Panizzon

Präsident

Dr. F. Gueissaz

Vorstandsmitglied 


\section{Assemblée générale extraordinaire de la Société Suisse de Dermatologie et Vénéréologie, 7 février 2002, Auditoire E.-Rossi, Inselspital Berne}

\section{Bienvenue}

A 9.40 h le président, Prof. Panizzon, ouvre la séance en saluant les 45(!) membres présents, et regrette que seuls 16 confrères aient pris la peine de s'excuser.

\section{Projet de réforme SSDV 2002 - Dr F. Gueissaz}

Le président Panizzon rappelle que c'est l'assemble générale de septembre 2001 qui a décidé de convoquer cette assemblée générale extraordinaire. Selon l'article 9 des statuts, les décisions sont prises par vote à main levée, à la majorité simple. Il n'est malheureusement pas possible de prendre en compte les votes par correspondance. Une volonté de modification du fonctionnement de notre SSDV était déjà dans l'air depuis plusieurs années, et le Prof. Panizzon remercie le Dr Gueissaz d'avoir concrétisé cette volonté. Le président signale que le Dr Gueissaz acceptera d'être candidat si son projet est accepté, et qu'il faudra modifier les statuts pour qu'il puisse accéder à cette présidence, arrivant au terme de 9 ans au comité.

Puis le Dr Gueissaz fait une introduction en allemand et présente brièvement son curriculum vitae, avant de reprendre en français pour une description de son projet, tel qu'il figure dans Dermatologica Helvetica. Le Dr Gueissaz veut en fait présenter un projet pour l'avenir de la SSDV et ne désire pas qu'il $\mathrm{y}$ ait d'amalgame entre son projet et sa candidature à la présidence. La gestion du temps est une préoccupation générale de notre époque, et le Dr Gueissaz estime que ceux qui en consacrent à la défense de nos intérêts communs méritent d'être dédommagés. Il estime par ailleurs que de très nombreux dossiers capitaux sont en cours et que la tâche s'annonce difficile. Il s'agit donc de rendre les postes au comité et à la présidence attractifs. Pour cela il envisage une rétribution correcte pour ceux qui travaillent, ainsi qu'un allégement des tâches de bureau, un meilleur suivi des dossiers, une aide pour le comité et pour les membres sous la forme d'un secrétaire général dont le profil précis reste à définir. Plusieurs interventions de l'assemblée suggèrent qu'il devrait s'agir d'un dermatologue (collègue travaillant à temps partiel, ou récemment retraité par exemple). L'idée du Dr Gueissaz est plutôt de rechercher un collaborateur avec une formation complémentaire et notamment de très solides connaissances de droit et d'économie. Il est évident que si cette personne a en plus une formation médicale ou mieux de dermatologue, cela le comblerait. Le Dr Gueissaz répond ensuite aux questions qui lui ont été adressées par écrit et remercie leurs auteurs. Il désire notamment insister sur le fait qu'il n'a jamais voulu faire de chantage entre la présentation de son projet et sa candidature à la présidence de la SSDV. Suivent de nombreuses questions et commentaires de la salle, notamment quant aux fonctions qui seraient déléguées au secrétaire général. Le Prof. Saurat suggère d'avoir plutôt une personne de formation «dermatologique», donc choisie dans nos rangs, et qui occuperait un poste de «chief executive officer». Dans le développement de ce concept, le Prof. Saurat pense que ce CEO devrait être élu par l'assemblée générale, et qu'il aurait ainsi une position plus claire et mieux définie dans l'organigramme de la société et des prises de décisions. Le président serait déchargé d'une quantité de tâches administratives et aurait alors le temps de la réflexion nécessaire à la présidence et à la prise de positions et de décisions.

Vote: A la question «Acceptez-vous le projet de réforme SSDV 2002 tel que présenté dans Dermatologica Helvetica, pour une durée de 3 ans?», le projet est accepté par oui: 35 voix - non: 8 voix - abstentions: 2 .

En vote complémentaire, on ajoute à ce projet: la préférence sera donnée à un candidat dermatologue ou avec une formation médicale. Ce point est accepté par oui: 19 voix - non: 17 voix - abstentions: 2 .

Il est donc décidé d'informer au plus vite tous les membres de la société dans le but de trouver dans nos rangs une ou un collègue intéressé par cette fonction.

\section{TarMed}

Le Dr Häuptli a accepté de venir faire une présentation du développement du TarMed et des projets pour le Reengineering 2. Celui-ci équivaudra à un important changement, mais dans l'ensemble, les modifications n'entreront en vigueur qu'en juin 2003, à part les cas d'urgence comme la radiologie. En fait, le TarMed n'est qu'un contrat entre des assureurs et des prestataires de soins, et qui pourra être modifié dans le temps. Il sera sous-tendu par la neutralité des coûts pendant environ 18 mois, en prenant comme base les chiffres des assurances de 2001, avec une petite pondération. Les 3 premiers mois seront «aveugles», c'est-à-dire que comme il n'y aura pas ou peu de factures il sera très difficile de savoir où l'on en est, puis viendra la phase de pilotage proprement dite où l'on apportera les modifications qui permettront de rester dans le cadre imposé. Puis viendra (dès 2003) la phase de «Tarifpflege», où l'on corrigera par l'introduction de nouvelles prestations et des modifications en fonction de l'évolution. Mais il faut s'imaginer une philosophie et une mécanique complètement nouvelle. En réponse à plusieurs questions concernant la pratique de notre spécialité, le Dr Häuptli répond que le tarif n'a pas pour but de régler notre pratique et qu'en fonction du principe des droits acquis, nous pouvons continuer notre pratique exactement comme jusqu'ici. Nous aurons ensuite 3 ans pour apporter les corrections par la Tarifpflege. La FMH s'efforce de sauvegarder les acquis de chaque spécialité, ceci également lors de conflits entre sociétés sur des «sujets frontières». Dr de Viragh s'étonne que des formations complémentaires engageant du temps et exigeant une formation supplémentaire ne permettent pas d'obtenir une meilleure rémunération que des prestations de base. Dr J.P. Grillet fait ensuite une présentation rapide de l'étude faite à Genève sur le TarMed dans la chirurgie ambulatoire, qui démontre un point pondéré de $\mathrm{CHF} 1,65$, avec une perte se chiffrant pour les chirurgiens (pour un point à $\mathrm{CHF}$ $1,-)$ se situant entre 40 et $60 \%$. Le Dr Häuptli signale qu'il n'a pas eu le temps de voir ces chiffres en détail et qu'ils vont à l'encontre de toutes les simulations faites dans les autres cantons. M. Häuptli convient que la situation est inquiétante et réfléchira à la réponse qu'il peut apporter à ces chiffres.

\section{FMS}

Plusieurs intervenants commencent en s'exprimant pour la sortie de la SSDV de la FMS, notamment au vu de l'absence de résultat de leurs actions en notre faveur que nous avons observé. Dr Brüngger propose plutôt de rediscuter avec eux, de 
voir ce qu'ils peuvent faire concrètement pour nous sur un an, avant de nous retirer. Dr H. Perroud insiste sur le fait que sans la FMS, la structure du TarMed serait encore plus mauvaise. Dr Bayard est aussi d'accord qu'il faut protéger notre activité opératoire et que le moment est mal choisi pour nous retirer. Pour le Dr Ramelet, c'est un placement à long terme que l'on va certainement pouvoir rentabiliser prochainement.

Vote: "Voulez-vous que la SSDV reste membre de la FMS au moins pour 1 an?» Accepté par oui: 38 voix - non: 6 voix abstentions: 1 .

Vote complémentaire: «Voulez-vous que la SSDV reste membre de la FMS pour 3 ans au moins?» Le projet est refusé par oui: 12 voix - non: 23 voix - abstentions: 6 .

\section{Varia}

Dr Gueissaz rappelle le délai d'inscription au 15 février 2002 pour le colloque de printemps à Expo02 avec un programme scientifico-récréatif très prometteur!

Avec cette annonce, le président Panizzon clôt cette assemblée générale extraordinaire à $12.30 \mathrm{~h}$.

Jean-Pierre Grillet

\section{Projet de modification des statuts (après l'assem- blée générale extraordinaire)}

\section{\$ 5 c Règlement d'indemnisation}

1) A partir d'un certain nombre d'heures, le travail effectué par les membres pour la société est indemnisé:

- le président de manière forfaitaire;

- les membres selon un décompte des heures de travail.

2) Les frais de voyage, d'hébergement et de repas sont indemnisés.

3) Tout membre susceptible d'être indemnisé en informe le trésorier au plus tard 2 mois avant l'assemblée générale pour que le budget puisse être établi.

4) Le montant des indemnisations est approuvé chaque année par l'assemblée générale dans le cadre du budget. Celleci décide également du nombre d'heures effectuées par les membres sans indemnisation.

5) Les notes de frais sont à adresser au trésorier de la société.

6) Les frais administratifs de secrétariat sont à la charge de la société.

\section{Base des indemnités pour 2003}

1) Indemnité du président CHF 30000,00

2) Indemnisation des activités des membres pour la société nécessitant la fermeture du cabinet, y compris le samedi
- une journée
CHF 1000,00
- une demi-journée
CHF 500,00
- une soirée
CHF 200,00

3) Indemnité de déplacement billet aller-retour première classe demi-tarif
4) Indemnité d'hébergement
$\mathrm{CHF}$
150,00 la nuit
5) Indemnité par repas
CHF 30,00
6) Nombre d'heures annuelles non indemnisées: $36 \mathrm{~h}$

\section{Secrétaire général de la SSDV}

L'assemblée générale extraordinaire de la SSDV du 7 février 2002 à Berne a accepté le principe d'un secrétaire général pour une durée de 3 ans.

\section{Procédure de nomination}

Une commission de nomination est constituée.

Cette commission est composée des 3 membres du bureau du comité de la SSDV et du candidat du comité au poste de président de la SSDV. didat.

La commission de nomination propose au comité au moins un can-

Le comité a la compétence de décision sur le choix de la personne.

A qualification égale, une préférence sera donnée à un membre de la SSDV.

Profil de la personne recherchée

- Formation universitaire, médicale, juridique ou économique

- Sens développé de la communication et de la négociation

- Facilité de rédaction et de communication en langue française et allemande

- Bonnes connaissances en informatique, Word, Excel, Powerpoint et Internet

- Autonome et flexible en matière d'horaires

Profil des tâches

- Responsabilité de l'administration de la société, des problèmes administratifs des membres (gestion des cotisations, etc.)

- Suivi des affaires courantes de politique professionnelle en collaboration étroite avec le président, le bureau et le comité

- Préparation et organisation des réunions du bureau et du comité

- Préparation et organisation des assemblées générales

- Préparation des bases de décisions pour le comité

- Mise en œuvre des décisions du bureau, du comité et de l'assemblée générale

- Valorisation du travail des différentes commissions permanentes de la SSDV

- Suivi de l'évolution de la politique de santé, propositions d'actions et de développement

- Création et maintien du contact avec la FMH et les autres sociétés de spécialistes

- Rédacteur des rubriques non médicales de Dermatologica Helvetica

- Rédacteur de la partie non médicale du site www.derma.ch

\section{Entrée en fonction}

1er novembre 2002

Révision des statuts (à prévoir après la période d'essai de 3 ans)

Article NN: Le secrétariat

Avec l'accord de l'assemblée générale, le comité peut confier diverses tâches administratives et de représentation à un secrétaire nommé en principe secrétaire général. Il est rétribué et a une voix consultative dans les décisions du comité. 


\section{Generalsekretär der SGDV}

Die ausserordentliche Generalversammlung der SGDV hat am 7. Februar 2002 in Bern das Prinzip eines Generalsekretärs für 3 Jahre angenommen.

Ernennung: Wahlprozedere

Eine Wahlkommission wird zusammengestellt.

Diese Kommission setzt sich aus dem dreiköpfigen Ausschuss der SGDV sowie aus dem vom Komitee vorgeschlagenen Präsidentschaftskandidaten zusammen.

Die Kommission schlägt dem Komitee einen Kandidaten vor.

Das Komitee hat die Entscheidungsbefugnis.

Bei gleichwertiger Eignung wird einem Mitglied der SGDV der Vorzug gegeben.

Profil der gesuchten Person

- Universitätsdiplom in Medizin, Jura oder Wirtschaft

- Sinn für Kommunikation und Verhandlungstaktik

- Sehr gute mündliche und schriftliche Kenntnisse der französischen und deutschen Sprache

- Gute Informatikkenntnisse in Word, Excel, Powerpoint und Internet

- Autonome und flexible Arbeitsweise

Aufgabenbereich

- Verwaltung der Gesellschaft sowie der administrativen Fragen ihrer Mitglieder (Verwaltung der Mitgliederbeiträge usw.)

- Verfolgen der Alltagsgeschäfte betreffend Berufspolitik in enger Zusammenarbeit mit dem Präsidenten, dem Ausschuss und dem Komitee

- Vorbereitung und Organisation der Versammlungen des Ausschusses und des Komitees
- Vorbereitung und Organisation der Generalversammlungen

- Vorbereitung der Entscheidungsgrundlagen für das Komitee

- Umsetzung der Entscheide des Ausschusses, des Komitees und der Generalversammlung

- Unterstützung der verschiedenen permanenten Kommissionen der SGDV

- Verfolgen der Entwicklung der Gesundheitspolitik und Handlungsvorschläge

- Herstellung und Pflege des Kontaktes zur FMH sowie zu den Fachgesellschaften

- Verfassen der nichtmedizinischen Rubriken in «Dermatologica Helvetica»

- Verfassen der nichtmedizinischen Beiträge auf der Website www.derma.ch

Arbeitsbeginn

1. November 2002

Revision der Statuten (ist erst nach der 3jährigen Probezeit vorzusehen)

Artikel NN: Das Sekretariat

Das Komitee kann mit Zustimmung der Generalversammlung grundsätzlich einen Generalsekretär mit verschiedenen Verwaltungsund Repräsentationsaufgaben beauftragen. Dieser wird entlöhnt und verfügt bei Entscheidungen des Komitees über eine konsultative Stimme. 